\title{
An Auditory Display to Convey Urgency Information in Industrial Control Rooms
}

\author{
Anna Sirkka, Johan Fagerlönn, Stefan Lindberg, and Ronja Frimalm \\ Interactive Institute Swedish ICT, Acusticum 4, \\ 94128 Piteå, Sweden \\ \{anna.sirkka, johan.fagerlonn, stefan.lindberg, \\ ronja.frimalm\} atii.se
}

\begin{abstract}
Auditory warning signals are common features in industrial control rooms. Finding sound signals that convey higher degrees of urgency while keeping the potential for annoyance low is challenging. In the present study, evaluations were performed on four different types of auditory displays. The displays were all designed to convey three levels of urgency. The examination focused on the following questions: (1) "How reliably can the operators identify the three levels of urgency?" and (2) "How annoying do the operators find the sound signals?". Fourteen operators participated in the study. For every signal within each auditory display, the participants were asked to rate the level of urgency and annoyance. The results show that one can design auditory displays that employ appropriate urgency mapping while the perceived annoyance is kept at a low level. The work also suggests that involving the end users in the design process could be advantageous.
\end{abstract}

Keywords: auditory display, control room, urgency, annoyance, warnings.

\section{Introduction}

Auditory warning signals are common features in many user environments, including vehicles, clinical facilities and industrial control rooms. Sound has certain advantages over other modes of interaction, especially in critical situations that require immediate attention. Salient auditory cues catch our attention; because hearing is omnidirectional, the sound can be perceived from any direction and wherever the operator has visual focus. Sound can provide information without adding visual load, which can be beneficial in demanding situations that require visual information processing (e.g., monitoring several process parameters on a display).

Nonetheless, the implementation of auditory warning signals is frequently careless. Edworthy [1] reports that the sound signals are too loud, too numerous and too confusing. Other authors have discussed the inappropriate use of auditory signals in a range of user contexts, including airplane cockpits and medical operating rooms [2, $3]$. In this study, we focus on the design of auditory warnings for industrial control rooms. The main objective is to develop auditory displays that assist operators effectively, while contributing to a better overall work environment. 


\subsection{Urgency Mapping}

Auditory warnings are designed to convey a sense of urgency. The term "urgency mapping" has been defined as matching the perceived urgency of a warning with the urgency of the threatening situation [4]. Appropriate urgency mapping is preferable, as it can help operators prioritize new information and minimize confusion. Inappropriate mapping may, however, have the opposite effect and potentially increase the workload. Therefore, a holistic approach in which all warnings in the operators' environment are considered according to the urgency mapping principle is essential to warning design. This approach is in accordance with the recommendations of the Engineering Equipment and Materials Users' Association (EEMUA), which states that an integrated design should be developed for all auditory warnings in a control room and that the operators' ability to identify the priority of the alarms is desirable [5].

Previous research has established that perceived urgency depends on the fundamental properties of the sound, including several spectral and temporal parameters [6-8]. By manipulating parameters such as speed and frequency content, a designer can systematically change the perceived urgency of the sound. Undoubtedly, learned associations could potentially "override" these mappings [9, 10]. However, considering the gains in learning time and reduced risk of confusion, adapting the physical characteristics from the very start is preferable.

\section{$1.2 \quad$ Annoyance}

Annoyance is an important characteristic to consider when implementing auditory warnings in any user context. In accordance with emotion regulation theory [11], operators may try to avoid experiencing the negative emotions associated with the sound simply by avoiding the sound. Considering that sound is omnidirectional and difficult to ignore, the only way to avoid the sound may be to turn the sound level down or to disable the function entirely. For instance, it has been reported that auditory warnings are frequently turned off in anesthetic operating rooms because of their unpleasant properties [3]. Furthermore, Wiese and Lee [12] reported that the annoyance of auditory warnings could be associated with increased workload levels.

There are many reasons why a sound can become an annoyance. Previous research shows that annoyance can be predicted based on physical and psychoacoustic parameters, such as loudness, sharpness duration and tonality [13-15]. For auditory warnings, it has been reported that increasing the urgency of the signal also increases the perceived annoyance [12, 16, 17]. Therefore, finding sounds that convey higher degrees of urgency while keeping the potential for annoyance low is challenging.

\subsection{Design of Warnings for a Control Room}

Warnings in different user contexts and situations demand different types of responses. For instance, collision warnings presented in a vehicle require an immediate response (e.g., the driver brakes). We argue that when urgent situations 
occur in a complex control room environment, it is generally essential that the operator remain calm and focus on solving the problem. The designers should attempt to find solutions that inform and guide the operator effectively and reliably while minimizing annoyance and disturbance. However, as described above, this task is challenging for the designer.

Although auditory warnings could be designed based purely on previous research results, in the present study, a user-centered design process is employed to find solutions that are more appropriate. Prior research provides an understanding of the parameters that influence urgency, annoyance, and distinguishability. However, the operators may also contribute knowledge regarding their work context, the type of urgent situations that can occur, and the task that needs to be performed. This additional insight can assist the designer in adapting the sounds to make them suitable and more tolerable in the work context.

\subsection{Aim}

In the present study, evaluations were performed on four types of auditory displays designed to assist operators in industrial control rooms. Each display was designed to convey three levels of urgency (low, medium and high). The examination focused on the following questions.

1. How reliably can the operators identify the three levels of urgency?

2. How annoying do the operators find the sounds?

Two of the evaluated concepts are referred to as Design 1 and Design 2. These auditory displays were designed with operators in a user-centered design process and were compared with two baseline displays to gain insight into the appropriateness of the solutions. Baseline 1 is currently in production and is delivered, along with solutions, from a control system manufacturer. Baseline 2 conveys different levels of urgency mainly by manipulating the frequency content of the sound.

\section{Method}

\subsection{Participants}

Fourteen control room operators, 1 female and 13 males, participated in the study. All subjects were employees of Smurfit Kappa Kraftliner Piteå. The mean age of the subjects was 46 years (SD 10). The mean experience of the subjects as operators was 22 years (SD 12). All subjects participated voluntarily. None of the subjects reported any hearing disorders relevant to the study. Originally, 15 operators performed the test but due to ambiguous answers, data from one test were excluded from analysis. 


\subsection{Apparatus}

The test took place in two different control rooms at Smurfit Kappa Kraftliner Piteå. Sounds were reproduced for the subjects using headphones (Philips HP890). The sounds were triggered through an application developed in Java and the test interface was presented on a laptop (Apple MacBook).

\subsection{Display Concepts}

Baseline 1. The Baseline 1 display uses a set of sound signals that is delivered along with solutions from a control system manufacturer. The signals are supposed to represent three levels of urgency. The signals are all abstract tonal sound signals, but they are quite different in character. The signals are used in their original form, i.e., as acquired from the manufacturer. The low-level warning consists of four tonal sounds, each with a length of approximately $580 \mathrm{~ms}$ and separated by approximately $580 \mathrm{~ms}$ of silence. The total length of the warning is approximately $4000 \mathrm{~ms}$. The mediumlevel signal consists of an approximately 2600-ms-long tonal sound. The high-level sound consists of two interpolating $250 \mathrm{~ms}$ tones with no separation (the first signal is only $75 \mathrm{~ms}$, but whether the signal is like that when implemented in the system is not clear). Figure 1 shows the FFT vs. time analysis for each signal. The sound levels presented are not the absolute values (levels as perceived by the participants). However, the relative differences are correct.

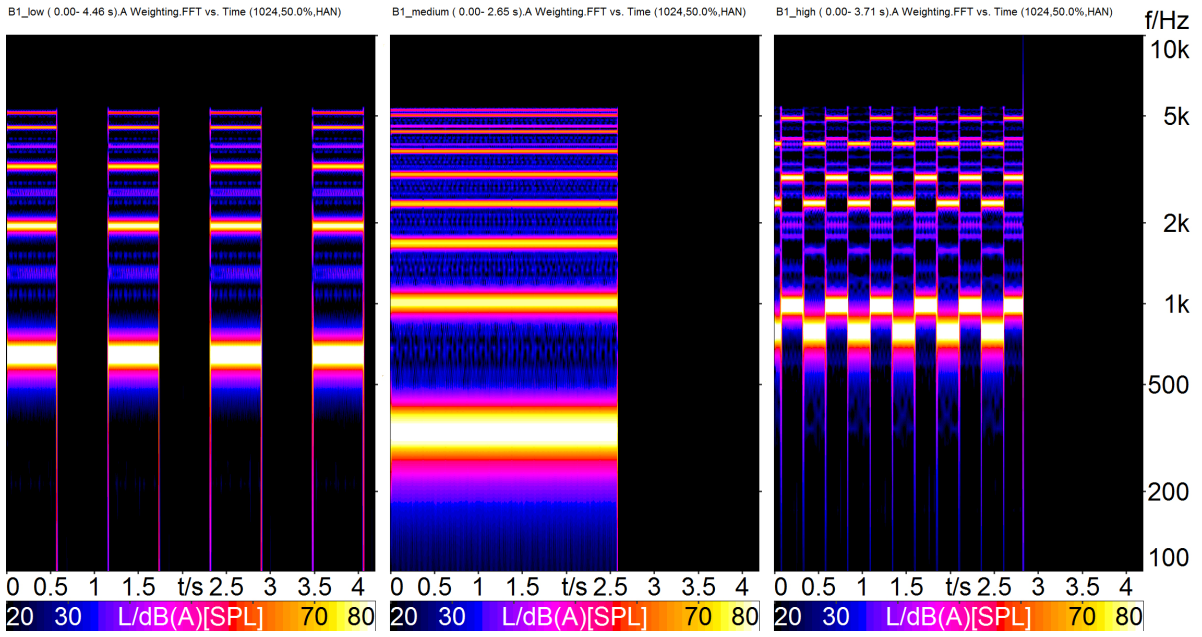

Fig. 1. FFT vs. time for the Baseline 1 sound signals

Baseline 2. Previous research has shown that increasing the fundamental frequency [6] or the amount of inharmonicity or dissonance [7, 18] of a sound increases the perceived urgency. The Baseline 2 display uses a combination of these parameters. The temporal properties were the same for all three signals. The signals consisted of 
five tonal sounds, each with a length of approximately $140 \mathrm{~ms}$ and separated by approximately $80 \mathrm{~ms}$ of silence (the last pause was approximately $50 \mathrm{~ms}$ making the signal sound like it was accelerating). The signals were constructed using the following sine tones: low urgency: $300 \mathrm{~Hz}$, medium urgency: $300 \mathrm{~Hz}+900 \mathrm{~Hz}+$ $3450 \mathrm{~Hz}$, high urgency: $300 \mathrm{~Hz}+2450 \mathrm{~Hz}+2550 \mathrm{~Hz}+3450 \mathrm{~Hz}+3513 \mathrm{~Hz}$. There was a slight difference in perceived loudness between the signals, with the lowurgency cue having the lowest level, followed by the medium-urgency and the highurgency signal. Figure 2 shows the FFT vs. time analysis for each signal within Baseline 2. The sound levels presented are not the absolute values, but the relative differences are correct.
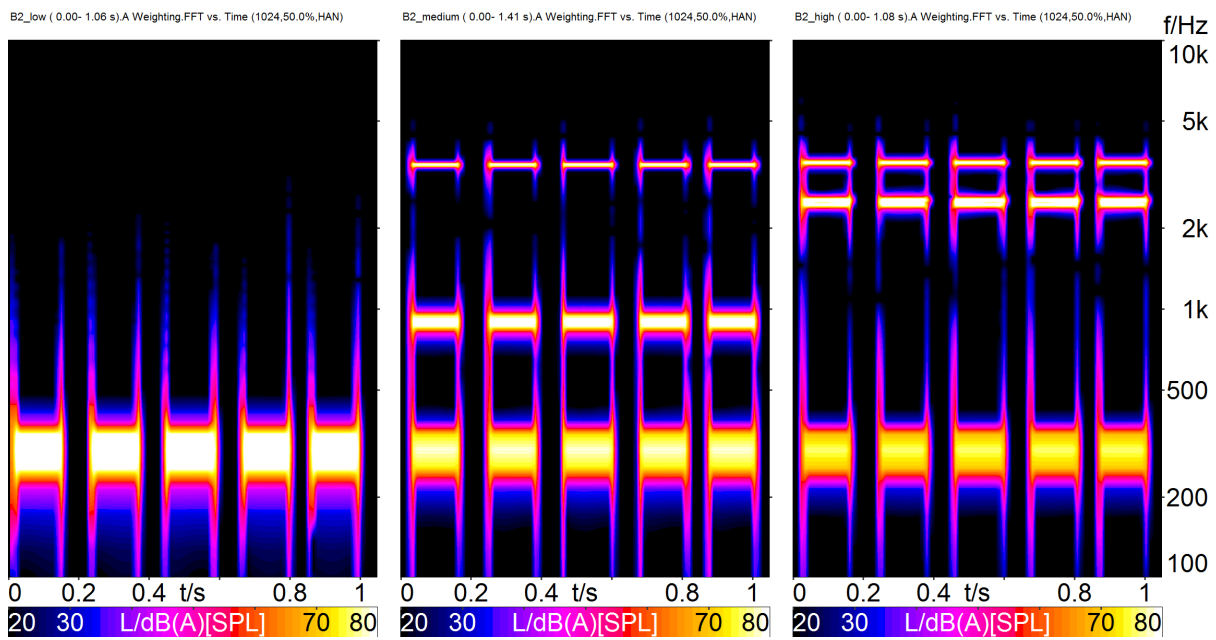

Fig. 2. FFT vs. time for the Baseline 2 sound signals

Design 1 and 2. Previous research has established that the number of repetitions, the speed, and the length of an auditory warning each affect the perceived urgency. Moreover, it has been suggested that the number of repetitions and the speed of a warning are more powerful in inducing changes in perceived urgency than a length change [19].

The Design 1 and 2 displays use a combination of the above-mentioned parameters. The displays have essentially the same temporal structure, where the lowurgency signals consist of one tonal sound with a length of approximately $500 \mathrm{~ms}$. The medium-urgency signals consists of two tonal sounds (total length: $1200 \mathrm{~ms}$ ) and the high-urgency signals are composed of three tones that are repeated twice (total length: $2300 \mathrm{~ms}$ ). The signals were subjectively assessed and adjusted in order to have approximately the same sound level.

The following notes comprise the Design 1 signals: low urgency: C4 (261.63 Hz), medium urgency: C4 $(261.63 \mathrm{~Hz})+\mathrm{D} 4(293.66 \mathrm{~Hz})$, high urgency: C4 $(261.63 \mathrm{~Hz})+$ D4 $(293.66 \mathrm{~Hz})+$ E4 $(329.63 \mathrm{~Hz})$. Figure 3 shows the FFT vs. time analysis for each signal. The sound levels presented are not the absolute values. 

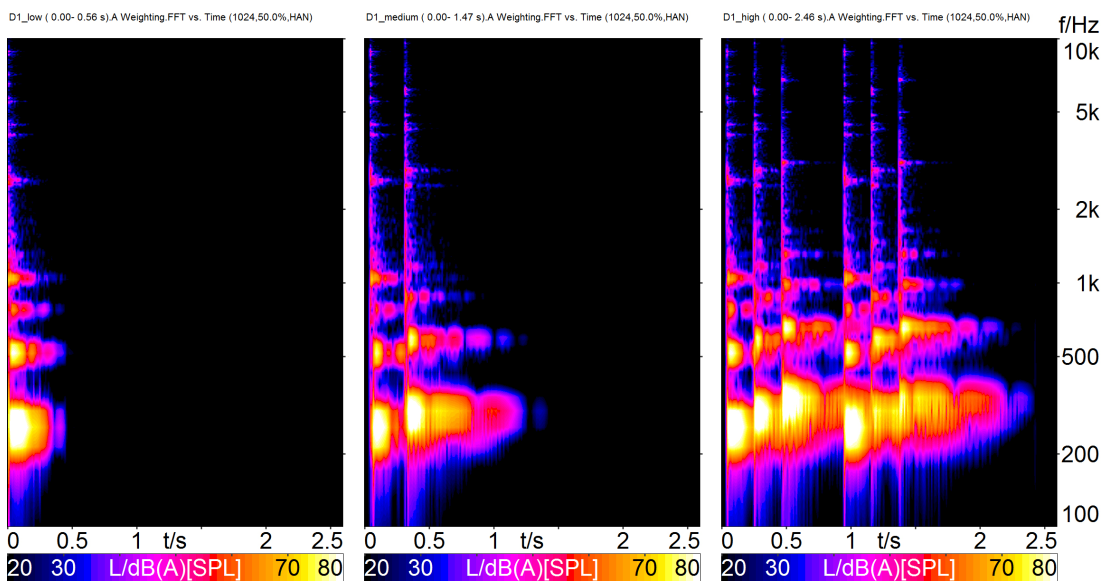

Fig. 3. FFT vs. time for the Design 1 sound signals

Design 2 uses the following notes: low urgency: $\mathrm{C} 4(261.63 \mathrm{~Hz})$, medium urgency: C4 $(261.63 \mathrm{~Hz})+\mathrm{F} 4(349.23 \mathrm{~Hz})$, high urgency: C4 $(261.63 \mathrm{~Hz})+\mathrm{F} 4(349.23 \mathrm{~Hz})+$ G4 $(392.00 \mathrm{~Hz})$. Figure 4 shows the FFT vs. time analysis for each signal. As with the previous analyses, the sound levels presented are not the absolute values.
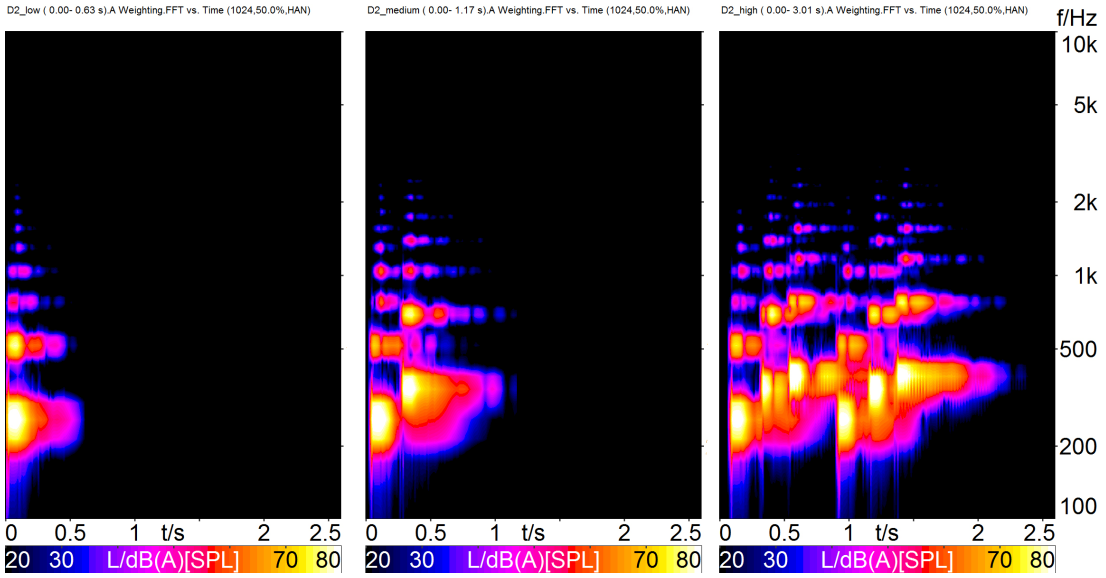

Fig. 4. FFT vs. time for the Design 2 sound signals

The character of the signals in Design 1 and 2 is based on acoustic musical instruments. Soft attacks, long decays, and natural harmonics were used with the aim of creating pleasant sounding signals. The signals of the Design 1 display have a marimba-like sound, and the signals in the Design 2 display are composed of a softsounding synth sound.

The Design 1 and 2 displays were developed through a user-driven design process that aimed to develop appropriate alarm sounds for control room environments. 
Twenty-four control room operators participated in the process. All subjects worked in the same control room at Smurfit Kappa Kraftliner Piteå. Operators participating in the design process were not used as test subjects during the listening test.

The process comprised an initial workshop followed by approximately ten design iterations. Each iteration consisted of two steps: the development of a design proposal and a user interaction. During a user interaction, a sound design proposal was presented to three to six operators. The design was discussed, and the feedback provided from the operators set the basis for the development of a refined design. Each interaction took place in the operators' own working environment, i.e., in a control room at Smurfit Kappa Kraftliner Piteå, and lasted for approximately half an hour.

\section{$2.4 \quad$ Procedure}

The subjects were seated at a table in front of a laptop computer in a remote part of the control room and received written instructions and a questionnaire. Both the instructions and the questionnaire were written in Swedish. The duration of the test was approximately 15 minutes.

The test contained four auditory displays. For each display, three sound signals, named A, B, and C, were judged. The participants listened to the sounds through an interface with three buttons representing signals $\mathrm{A}, \mathrm{B}$, and $\mathrm{C}$. The perceived sound levels of the four auditory displays were subjectively adjusted to be approximately the same.

The level of the sound signals was subjectively adjusted to be clearly audible in the present background noise. The subjects could listen to the sound signals of each auditory display as many times as they wished.

To reduce order effects it was desirable that each display was presented first an equal number of times. Therefore the first display to be assessed was specifically chosen prior to testing. The order of the following three displays was randomized, as were the signals within each display. Due to drop-outs the Baseline 2 display initiated a test only twice. The other three displays initiated a test four times each.

For each auditory display, the participants were asked to rate the level of urgency on a stepless scale ranging from "low" to "high". The Swedish word "prioritet" is used by the operators themselves to grade warnings and was used in this study to represent urgency. Similarly, the operators were asked to rate the level of annoyance on a scale ranging from "not at all" to "much". There was also an open comments section for each auditory display.

\subsection{Dependent Variables}

Ratings of urgency and annoyance are two dependent variables of the evaluation. Additionally, the number of subjects that successfully estimated the correct order of urgency (for all three sounds in the display) is a dependent variable. 


\section{$3 \quad$ Results}

\subsection{Urgency}

Figure 5 shows the results of the urgency rating for the sound signals in each display type. All displays, except Baseline 1, resulted in mean ratings indicating an appropriate urgency mapping.

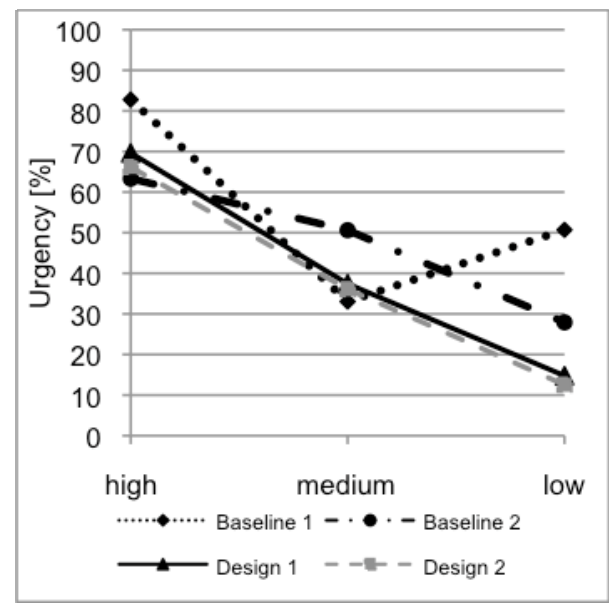

Fig. 5. Mean urgency ratings $(100 \%=$ high urgency)

A one-way ANOVA, followed by post-hoc analysis (Tukey's HSD test), showed that, for Design 1 and 2, each signal in the display differed significantly from the other two $(\alpha=0.05)$. For Baseline 1, the differences between sound signals were significant, but the low-level signal was rated more urgent than the medium-level signal. For Baseline 2, the difference between the medium- and high-level signals was not statistically significant.

Figure 6 shows how many subjects successfully estimated the (intended) urgency levels for the auditory displays. For Design 1, all participants rated the urgency levels correctly, while for Baseline 1, only two subjects rated the urgency levels as intended. 


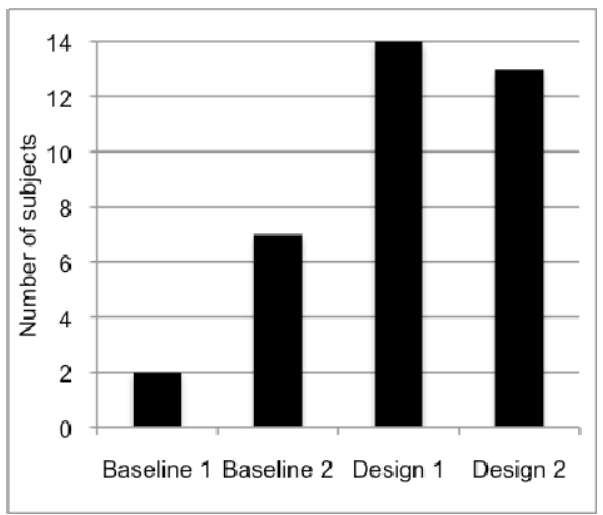

Fig. 6. The number of successful urgency estimations

\subsection{Annoyance}

Figure 7 shows the mean annoyance ratings for each display type. As expected, sound signals were rated more annoying for the higher urgency levels. However, the signals in Design 1 and 2 displays received low or intermediate mean annoyance scores, while the signals in the baseline displays received intermediate or high mean scores.

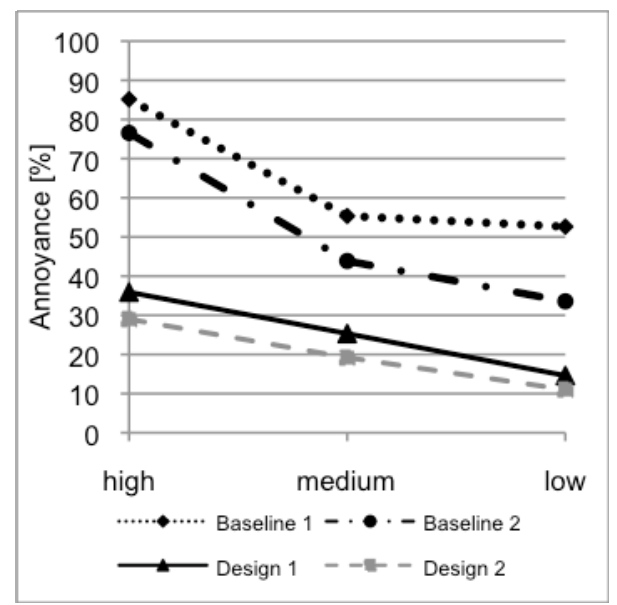

Fig. 7. Mean annoyance ratings $(100 \%=$ much $)$

\section{Follow-Up Study for Baseline 1}

During the evaluation, it was realized that the lengths of the Baseline 1 signals varied. The length of an auditory warning may affect the perceived urgency [19]. For Baseline 1, the low-urgency signal was rated more urgent than the medium-urgency signal. As the low-urgency signal was approximately $1400 \mathrm{~ms}$ longer than the 
medium-urgency signal (low=4000 ms, medium=2600 ms), a follow-up study was conducted in order to find out whether this difference in length could be the reason for the incorrect urgency mapping.

Fourteen subjects (not control room operators), 6 female and 8 males, participated in the follow-up study. The mean age of the subjects was 35 years (SD 14). All subjects participated voluntarily. None of the subjects reported any hearing disorders that would be of relevance for the study.

The test took place in a desktop environment and the procedure was the same as in the main study. The signals within the display were randomized and participants were asked to rate the level of urgency of the Baseline 1 signals. Consequently, ratings of urgency constituted one dependent variable of the evaluation. Additionally, as in the main study, the number of subjects that successfully estimated the correct order of urgency constituted a dependent variable.

The mean values for the low-urgency and medium-urgency signals were $41.4 \%$ and $43.0 \%$, respectively (where $100 \%$ is representing high urgency). A one-way ANOVA, followed by post-hoc analysis (Tukey's HSD test) showed no significant differences between the two signals. Apart from that, the differences were significant $(\mathrm{p}<0.01)$. Five subjects rated the urgency levels as intended.

\section{Discussion}

How reliably can the operators identify the three levels of urgency?

The results support that, for the Design 1 and 2 displays, operators can reliably identify the three levels of urgency. For Design 1, all participants judged the urgency levels as intended. The sounds used a combination of spectral and temporal parameters to express different urgency levels. We cannot draw any conclusions regarding individual parameters and their impact on perception, but taken together, the combination of parameters and their levels resulted in two very promising display designs.

In the Design 1 and 2 displays, the high-urgency sounds received relatively low mean scores. These results are not surprising considering the non-intrusive design of the signals. Auditory warnings can definitely be shaped to sound more urgent by manipulating the sound parameters to more extreme levels. However, we argue that it is essential that the operators can easily and reliably distinguish between urgency levels. However, low levels of perceived urgency may be of importance and cause confusion in user environments where the warnings occur less frequently and where operators do not have the chance to learn the meanings of the sound signals.

The results of the present work show that the signals in Baseline 1 (which is currently delivered by a control system manufacturer) employ an inappropriate urgency mapping. The main study indicates that switching the low- and mediumurgency signals would result in a better mapping. The follow-up study (which tested sounds of equal length) did not support that either of these two signals is perceived as more urgent than the other. For the Baseline 2 display, the differences in scores between signals were not particularly large and the difference between the high- and 
medium-urgency signals was not statistically significant. The combination of sound parameters and the selected levels seem to be insufficient in making the sound signals distinguishable in terms of urgency.

How annoying do the operators find the sound signals?

The operators judged none of the signals in the Design 1 and 2 displays to be particularly annoying. Even the high-urgency signals received low to intermediate mean scores as opposed to the high-urgency signals in the Baseline 1 and 2 displays, which received very high annoyance ratings. The rather low annoyance ratings observed for the Design 1 and 2 displays support the appropriateness of these solutions. We cannot draw any conclusions regarding individual sound parameters and their impacts on annoyance. Thus, we cannot make any statements regarding exactly what made the signals in the Baseline 1 and 2 displays more annoying.

A system manufacturer currently delivers the sound signals used in Baseline 1 . Conclusions regarding the appropriateness of these signals based on the annoyance ratings observed in the present study should be made with caution. One parameter that might influence annoyance levels is the duration of the signal (a longer duration might be more annoying). The sound signals used in Baseline 1 had longer durations than the signals in the other displays (which may have contributed to higher annoyance levels). However, the extent to which the selected durations for the Baseline 1 signals represent "typical" durations when the signals are implemented in real control room settings was not investigated.

In conclusion, the results support Display 1 and 2 as appropriate auditory displays to convey urgency information to control room operators. The work also support that auditory warning displays can be designed to employ appropriate urgency mapping while keeping the perceived annoyance of the sound signals at a low level. In real implementations, the annoyance of alarms may depend on a range of factors (alarm frequency, false alarm frequencies, etc.) that were not investigated in the present study. Still, the results support that it is worthwhile for system and sound designers to try to lower the perceived annoyance levels of control room alarm sounds. A sound signal that is both effective and has non-annoying characteristics is more likely to become tolerable. Finally, the present work suggests that involving the end users in the design process could be advantageous in reaching successful auditory display solutions.

Acknowledgments. Research presented in this paper was funded by EU:s Structural Funds, the County Administrative Board of Norrbotten, Piteå Municipality, Skellefteå Municipality, and RISE.

\section{References}

1. Edworthy, J.: The Design and Implementation of Non-verbal Auditory Warnings. Applied Ergonomics 25(4), 202-210 (1994)

2. Ulfvengren, P.: Design of Natural Warning Sounds. In: Scavone, G.P. (ed.) Proceedings of the 13th International Conference on Auditory Display, Schulich School of Music, McGill University, Montreal, pp. 146-153 (2007) 
3. Block, F.E., Nuutinen, L., Ballast, B.: Optimization of Alarms: A Study on Alarm Limits, Alarm Sounds, and False Alarms, Intended to Reduce Annoyance. Journal of Clinical Monitoring and Computing 15, 75-83 (1999)

4. Edworthy, J., Adams, A.: Warning Design: A Research Perspective. Taylor \& Francis Ltd., London (1996)

5. Engineering Equipment and Materials Users' Association (EEMUA): Alarm Systems - A Guide to Design, Management and Procurement. EEMUA, London (2007)

6. Edworthy, J., Loxley, S., Dennis, I.: Improving Auditory Warning Design: Relationship between Warning Sound Parameters and Perceived Urgency. Human Factors 33(2), 205$231(1991)$

7. Hellier, E.J., Edworthy, J., Dennis, I.: Improving Auditory Warning Design: Quantifying and Predicting the Effects of Different Warning Parameters on Perceived Urgency. Human Factors 35(4), 693-706 (1993)

8. Haas, E.C., Edworthy, J.: Designing Urgency Into Auditory Warnings Using Pitch, Speed and Loudness. Computing \& Control Engineering Journal 7(4), 193-198 (1996)

9. Guillaume, A., Drake, C., Rivenez, M., Pellieux, L., Chastres, V.: Perception of Urgency and Alarm Design. In: Nakatsu, R., Kawahara, H. (eds.) Proceedings of the 8th International Conference on Auditory Display, pp. 357-361. Advanced Telecommunications Research Institute, Kyoto (2002)

10. Burt, J.L., Bartolome, D.S., Burdette, D.W., Comstoc, J.R.: A Psychophysiological Evaluation of the Perceived Urgency of Warning Signals. Ergonomics 38(11), 2327-2340 (1995)

11. Gross, J.J.: Emotion Regulation in Adulthood: Timing is Everything. Current Directions in Psychological Science 10(6), 214-219 (2001)

12. Wiese, E.E., Lee, J.D.: Auditory Alerts for In-vehicle Information Systems: The Effects of Temporal Conflict and Sound Parameters on Driver Attitudes and Performance. Ergonomics 47(9), 965-986 (2004)

13. Hiramatsu, K., Takagi, K., Yamamoto, T., Ikeno, J.: The Effect of Sound Duration on Annoyance. Journal of Sound and Vibration 59(4), 511-520 (1978)

14. Khan, M.S., Johansson, Ö., Sundback, U.: Development of an Annoyance Index for Heavy-duty Diesel Engine Noise Using Multivariate Analysis. Noise Control Engineering Journal 45(4), 157-167 (1997)

15. Landström, U., Åkerlund, E., Kjellberg, A., Tesarz, M.: Exposure Levels, Tonal Components and Noise Annoyance in Working Environments. Environment International 21(3), 265-275 (1995)

16. Marshall, D.C., Lee, J.D., Austria, P.A.: Alerts for In-vehicle Information Systems: Annoyance, Urgency and Appropriateness. Human Factors 49(1), 145-157 (2007)

17. Tan, A.K., Lerner, N.D.: Multiple Attribute Evaluation of Auditory Warning Signals for In-vehicle Crash Avoidance Systems. Technical Report, National Highway Traffic Safety Administration (1995)

18. Russo, F.A., Lantz, M.E., English, G.W., Cuddy, L.L.: Increasing Effectiveness of Train Horns Without Increasing Intensity. In: Brazil, E., Shinn-Cunningham, B. (eds.) Proceedings of the 9th International Conference on Auditory Display, pp. 51-54. Boston University Publications Production Department, Boston (2003)

19. Hellier, E., Edworthy, J.: Quantifying the Perceived Urgency of Auditory Warnings. Canadian Acoustics 14(4), 3-11 (1989) 